

\section{Student Paramedics Australasia Conference Posters}

\section{List of presenting authors:}

\section{Alicia Armstrong}

The University of the Sunshine Coast

a_a006@student.usc.edu.au

\section{Tabitha Broome}

Edith Cowan University

tbroome87@outlook.com

\section{Georgia Melville}

Ambulance Service of New South Wales

gmelville@ambulance.nsw.gov.au

Rachel Kluck (winner of competition)

The University of Queensland

rachel.kluck@uqconnect.edu.au

\section{David Still}

Australian Catholic University dmstil001@myacu.edu.au

\section{Sarah Lightowler}

Queensland University of Technology

sarah.lightowler1@gmail.com 


\section{The Good and the Bad of Adrenaline in Out of}

\section{Hospital Cardiac Arrests: Could we make it better?}

\section{Alicia Armstrong, University of the Sunshine Coast, 2nd Year Student}

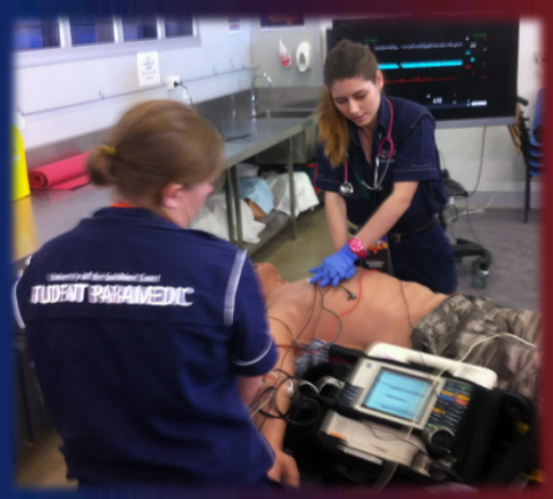

(contact number 0417647 865)

\section{Background}

Adrenaline is used frequently in out of hospital cardiac arrests with most ambulance services including the administration of adrenaline in their cardiac arrest protocols. Though current evidence is undecided on the role of adrenaline in Out-of-Hospital Cardiac -Arrests (OHCA) and its long term effects on patient survival.

\section{Method}

Looking at the positive and negative effects of how adrenaline works in the setting of out of hospital cardiac arrest and looking at research that could make adrenaline more targeted so only the positive effects are achieved, was the main research undertaken to gain a greater understanding of the topic.

\section{Conclusion}

Most of the studies indicate that adrenaline helps with short term survival, but not with long term survival or favourable neurological outcomes. The detrimental effects of adrenaline on a heart was briefly explored. This report also looked at how adrenaline works on both $\alpha$ and $\beta$ receptors and that the use of $\beta$ blockers could be an area of research into the future.

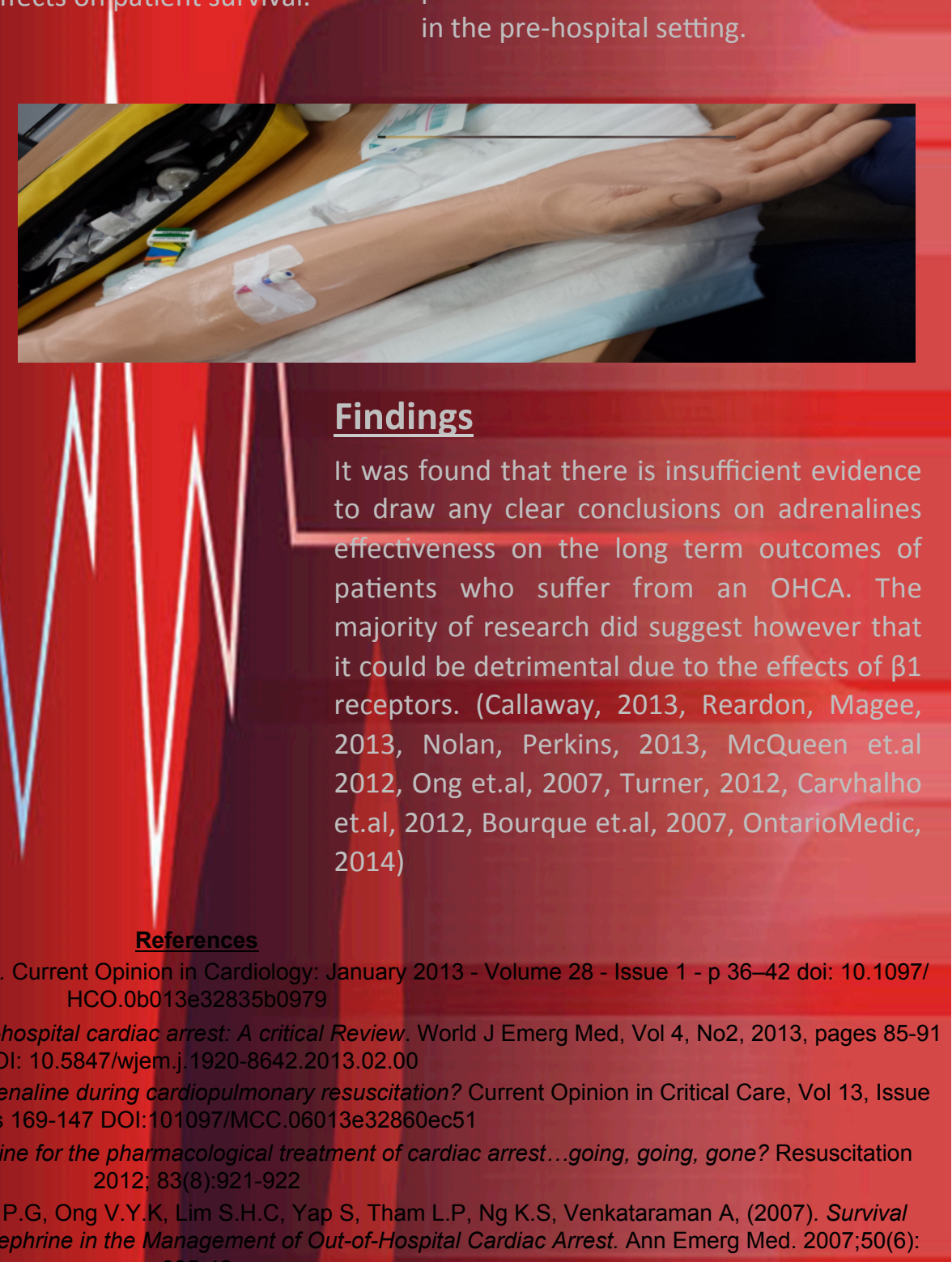

\section{Objective}

This was written to explore the efficacy of adrenaline in OHCA, looking at both positive and negative effects of adrenaline. Also the idea that by using Beta $(\beta)$ Blockers in conjunction with adrenaline, the paramedic may be able to negate some of the negative effects and increase long term survivability in patients who do suffer a cardiac arrest in the pre-hospital setting. 
Ectopic Pregnancy Sites

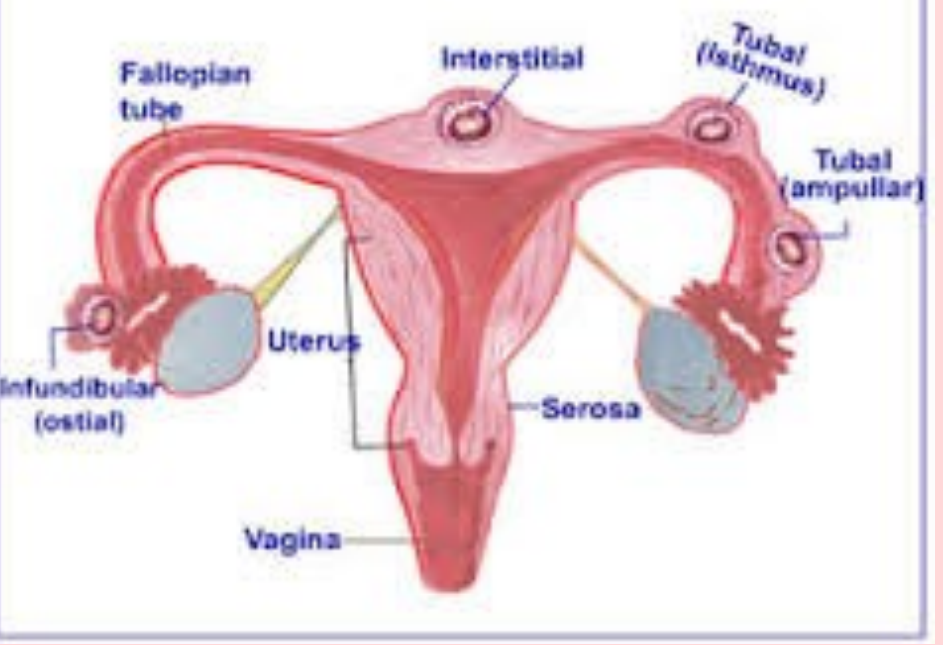

Image 1

By: Tabitha Broome. 3rd Year ECU Student-WA

\section{Background}

An ectopic pregnancy can be defined as the implantation of a fertilised ovum outside the uterine cavity (Houry \&Keadey, 2007). Mistovich and Karren (2010) expand on this by including places of possible implantation such as the fallopian tube, the abdominal peritoneal covering, the outside wall of the uterus or cervix. Most ectopic pregnancies implant in the fallopian tube at a rate of $98 \%$ (Jurkovic \& Wilkinson, 2011). The expected pathway for early pregnancy involves the fertilised ovum travelling along the fallopian tube via the peristaltic action of the cilia located in the lining of the tube (Marieb \& Hohen, 2010). The blastocyst then implants itself on the wall of the uterus and the foetus develops (Pairman, Pincombe, Thorogood \& Tracy, 2006). An ectopic pregnancy occurs due to a delay in passage from the fallopian tube to the uterus. This delay can be caused by a tubal infection that destroys the cilia, tubal ligation or previous tubal surgeries (Pairman et al., 2006). Instead the blastocyst implants itself in the epithelium of the fallopian tube and the foetus develops causing a tubal pregnancy. This can either rupture at 5-7 weeks or be expelled from the tube into the peritoneal cavity at 8-10 weeks causing a tubal abortion (Pairman et al., 2006).

\section{Objective}

The purpose of this literature review was to discover what an ectopic pregnancy is and the pre hospital interventions and management guidelines for ectopic pregnancy within Australia.

\section{Method}

Search terms: "Ectopic" "Pregnancy" "Pre-hospital" "Management" "Interventions" "Clinical Practice Guidelines" "Australia" "Medical Emergencies"

Search Engines: Library One (ECU Library), Medscape, EMSWorld, Canadian Medical Journal

Books: Mosby's Paramedic Textbook, Human Anatomy \& Physiology Pre-hospital Emergency Care, Midwifery Preparation for Practice

\section{The Review}

\section{Key Risk Factors}

A group of experts agree (Navarro, 2009; Sanders, 2012; Houry \& Keadey, 2007) any female of child bearing age, usually between 25-34 years, is at risk for an ectopic pregnancy. These experts also agree that this risk is increased in older females and minorities. Many more experts (Mistovich \& Karren, 2010; Murray, Baakdah, Bardell \& Tulandi, 2005) agree with these risk factors and expand on them by including patients with previous tubal pregnancies, previous tubal surgeries or tubal ligation, patients with a history of pelvic inflammatory disease, history of infertility and patients with a history of sexually transmitted infections. The use of the intrauterine device (IUD) as a contraceptive will decrease the risk of pregnancy however, if a patient falls pregnant with an IUD their risk of ectopic pregnancy increases (Houry \& Keadey, 2007).

\section{Pre-Hospital Management}

Navarro (2009) reminds paramedics of two important principles in managing ectopic pregnancy. These are; definitive care is not always possible in the field; and caring for the mother is caring for the foetus. Houry and Keadey (2007) expand on this first point by explaining that the role of the paramedic is to stabilise the patient, insert two large bore IV gauges and transport to hospital. A large group of experts (Navarro, 2009; Sanders, 2012; Mistovich \& Karren, 2010; Snyder, Kivlehan \& Collopy, 2012; Greenwald \& Keadey, n.d.). agree that management of the female patient, within child bearing years and presenting with possible abdominal pain and vaginal bleeding, should include; high flow oxygen therapy via a nonrebreather mask, analgesia, position of comfort and transport. This should be coupled with a high index of suspicion for ectopic pregnancy and an informative patient history.

Questions to ask during a patient history include the last date of menstruation, whether the pregnancy is known and if the patient has had any other pregnancies (Greenwald \& Keadey, n.d; Navarro, 2009). Paramedics should take a full gynaecological and obstetric history including who is administering their prenatal care, is there a presence of vaginal bleeding, when is the delivery date and how many weeks pregnant the patient is (Navarro, 2009; Greenwald \& Keadey, n.d.). Sanders (2012) also suggests that questions should be asked in a safe environment, away from relatives or partners as the patient may have information they do not want others to know.

Pain in ectopic pregnancy can be diffuse, cramp and non-specific (Snyder et al., 2012). In a ruptured ectopic pregnancy pain can be described as sharp, severe, constant and peritoneal in nature (Snyder et al., 2012). Sanders (2012) describe the signs and symptoms as severe abdominal pain which may radiate to the neck or shoulder whilst Mistovich and Karren (2010) describe the pain as a dull ach or knife-like.

Paramedics are often the first medical intervention patients receive. Due to a lack of equipment and specialised expert training, paramedics are unable to diagnose abdominal conditions that a patient may present with. This lack of visibility of the patient's abdominal cavity should cause paramedics to have a high index of suspicion of ectopic pregnancy in female patients of child bearing years (Sanders, 2012). The female patient with abdominal pain, who calls the paramedics, may not know she is pregnant. A delay in the diagnosis of pregnancy may be due to the patient still menstruating while she is pregnant, a patient being asymptomatic of pregnancy or the patient may not be of socially accepted child bearing years.

Pre-hospital care of ectopic pregnancy is limited and a delay in considering an ectopic pregnancy in the differential diagnosis can result in an adverse outcome for the patient. A major complication of this condition is a ruptured ectopic pregnancy. Paramedics must be prepared to manage shock as a result of the ruptured pregnancy. In the presence of haemorrhage, shock may not be noticeable until the patient has lost approximately $40 \%$ of her circulating blood volume. Compensatory mechanisms of the mother are activated during haemorrhage to protect the foetus and mother (Navarro, 2009). This is a true medical emergency as the patient can be in pain, suffering from a haemorrhage either through the vaginal canal or into the abdominal cavity. Haemorrhage, if significant enough, can lead to hypovolaemic shock. Sanders (2012) insinuates that the management of this condition requires initial resuscitation and rapid transport to hospital for a surgical intervention.

Navarro (2009) reminds the reader that caring for the mother is caring for the foetus. As the pregnancy is less than 20 weeks into gestation, it is a non-viable pregnancy and all care must be directed towards the mother (Pairman et al., 2006). Queensland Ambulance Service clinical practise guidelines (QAS CPG) suggest that the initial care should include assessment of the ABC's and the protocols for abdominal pain management. If the patient is in shock QAS CPG recommend that paramedics follow the shock management protocols. If the patient is not in shock, the QAS (2011) recommends that paramedics consider analgesia, anti-emetics and intra-venous fluid be administered. Notification to the receiving hospital and transport to that hospital conclude the QAS (2011) recommendations for ectopic pregnancy treatment. Research into St John Ambulance Northern Territory and Ambulance Victoria guidelines proves that there are no specific guidelines for ectopic pregnancy in these services.

\section{Conclusion}

As paramedics can only treat what they see, emergency medical responders will only ever treat a "suspected rupture of ectopic pregnancy". The confirmed diagnosis will be made in the hospital using their diagnostic equipment. When treating abdominal pain in a female patient of child bearing age, paramedics are required to have a high index of suspicion of ectopic pregnancy and gather an informative patient history. Prior notification and rapid transport to hospital is required for patients, especially those who have life threatening symptoms.

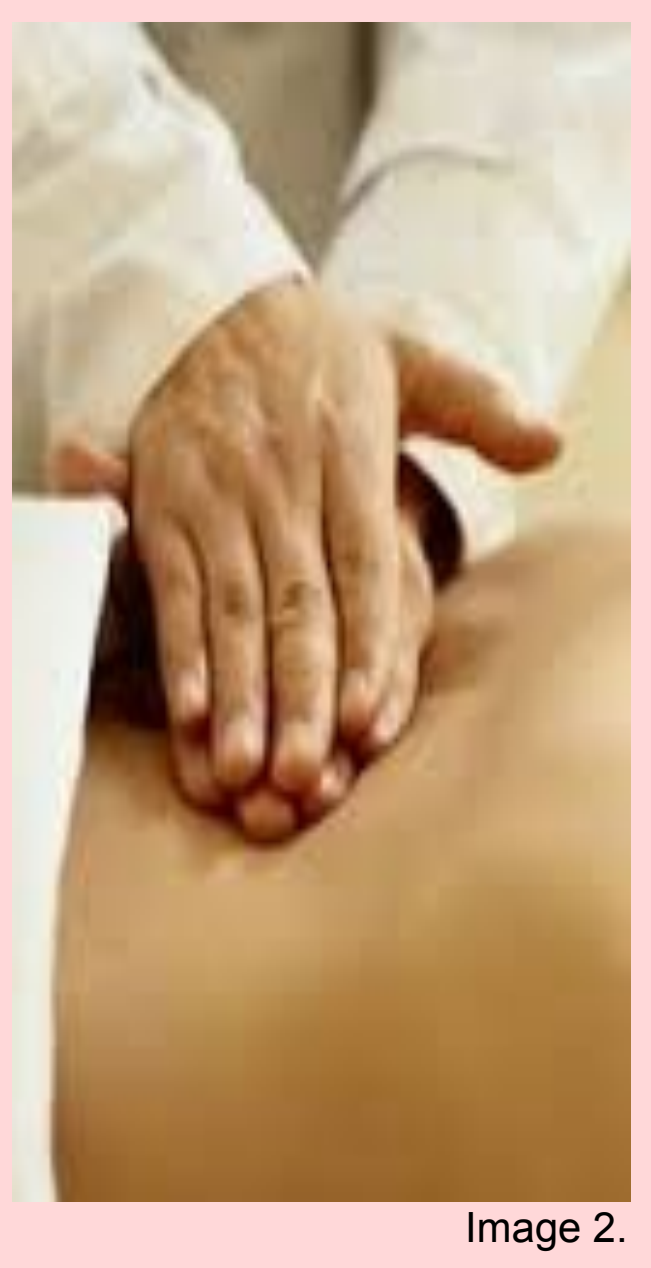

References

Greenwald, I. B., \& Keadey, M. T. (n.d). Obstetric and gynecologic emergencies. Retrieved August 01, 2013, from Emergency Medicine: http:// MEI5U0x6VkR6cHhpTVgwUIVNV0pHZEZGaE9VVQ\%3D\%3D_0.pd Houry, D., \& Keadey, M. (2007). Complications in pregnancy part 1: early pregnancy. Emergency Medicine Practise, 9(6), 1-28.

Image 1. Retrieved from: http://www.medindia net/drugs/medical-condition/

Image 2. Retrieved from: http://www.pregnancypregnant.org/abdominal-examination-inpregnancy-step-by-step-guide.htm

Jurkovic, D., \& Wikinson, H. (2011). Diagnosis and management of ectopic pregnancy. BMJ, 1-6.

Marieb, E. N., \& Hoehn, K. (2010). Human anatomy \& physiology (8th ed.). San Fransico, USA: Pearson.

Mistovich, J. J., \& Karren, K. J. (2010). Prehospital emergency care (9th ed.). Saddle River, New Jersey, America: Pearson Education Inc.

. Bardell, T. \& Tulandi, T (2005). Diagnosis and treatment of ectopic pregnancy. Canadian Medical Association Journal, 173(8), 905-912.

Navarro, K. (2009). Prehospital management of obstetric complications. Texas EMS Maga-

zine, 32-39.
Pairman, S., Pincombe, J., Thorogood, C., \& Tracy, S. (2006). Midwifery preparation for practice. Marrickville, Australia: Elsevier

Queensland Ambulance Service. (2011). Obstetrics - clinical practice guidelines. Retrieved

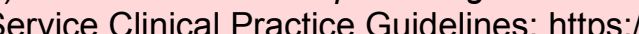
ambulance.qld.gov.au/medical/pdf/05 cpg obstetrics.pdf

Sanders, M. J. (2012). Mosby's paramedic textbook (4th ed.). Missouri, USA: Elsevier. Snyder, S., Kivlehan, S., \& Collopy, K. (2012, November 1). Vaginal bleeding in the pregnant patient. Retrieved August 6, 2014, from EMSWorld: http://www.emsworld.com article/10811951/vaginal-bleeding-in-the-pregnant-patient 
4 $50 \%$ of patients with Hyperkellente present with no ECG jelatided

ohanges eVen with potassiun LeVels greater thear

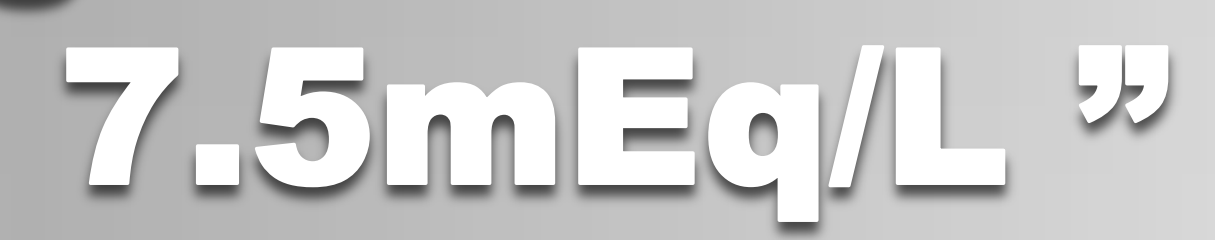

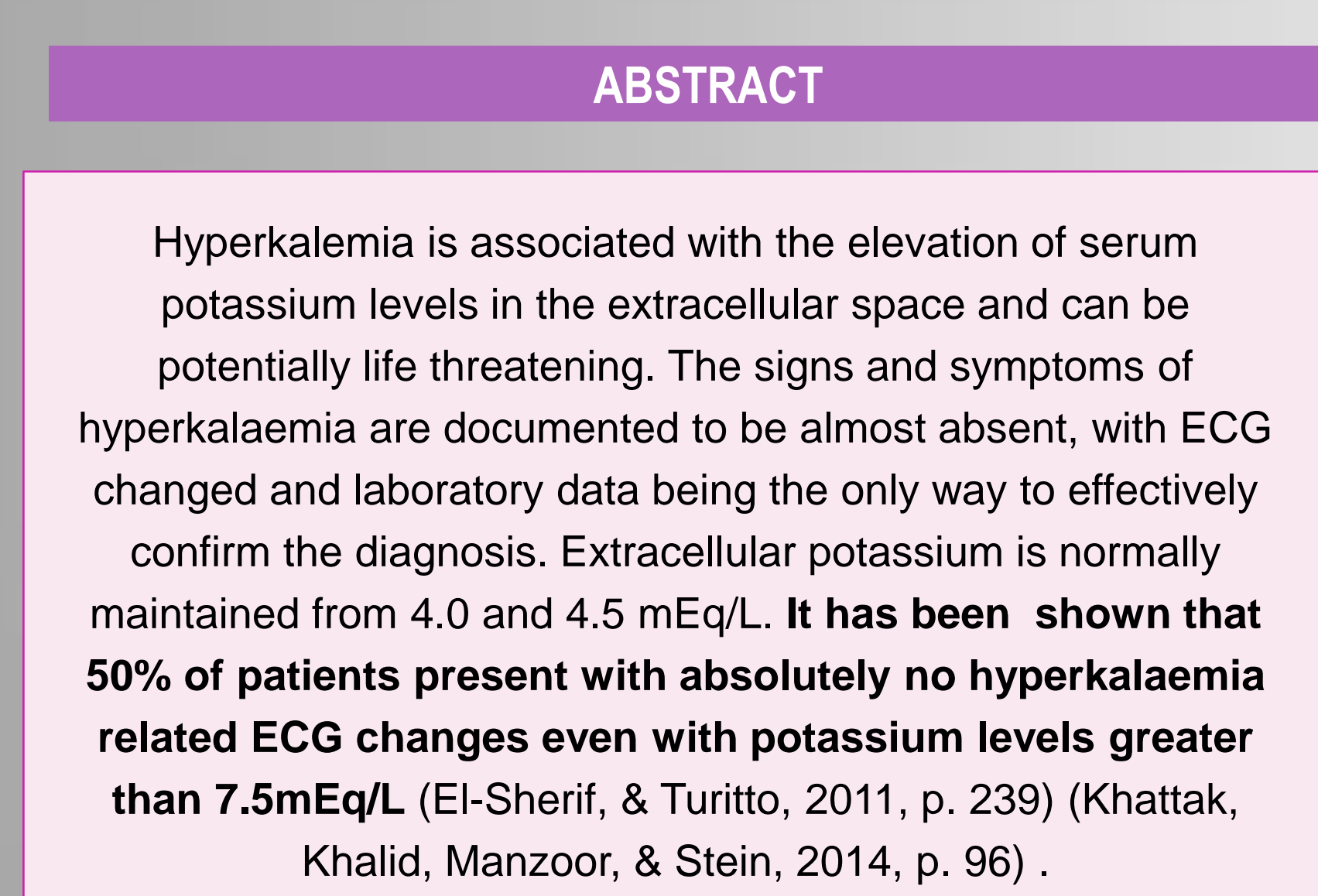

So where does this leave Paramedics? Clinically, if all Paramedics can only base their diagnosis on ECG related changes and history, does this give weight to doing pre- hospital point of care blood potassium level testing?

\section{HYPERKALEMIA- Think normal ECG!} GEORGIA MELVILLE- Paramedic Student Charles Sturt University
Hyperkalaemia = Cardiac Death

When potassium levels do increase, this causes a shift in the resting membrane potential of cells $(-90 \mathrm{mV})$ due to the influx of potassium. This causes slowing of conduction through the myocardium and a prolongation of membran depolarisation. Only $50 \%$ of patients experiencing this will have progressive ECG changes which are displayed in progression strip below

Causes of hyperkalemia

Renal Failure

Liver disease Heart failure Medications:
Burns

Excessive use of dietary salts (potassium chloride) Crush injuries Chemotherapy Metabolic Acidosis Dialysis
Dicis Addison's disease Hyperkalaemia periodic paralysis Point Of Care Testing - (POCT)

Ahn, et al. $(2011$, p. 25) has shown that POCT using a blood gas analyser compared to central laboratory testing (CLT) gives a mean value which is $0.19 \mathrm{mmo} / /$ different. In accordance with the United States Clinical laboratory Improvement Amenament, a difference of up to $0.5 \mathrm{~m}$ mol/ in measure potassium between CLT Fleming, \& Selvakumar, 2011, p. 24). This shows that Fleming, \& Selvakumar, 2011, p. 24). This shows that POCT is a valuable, cases wh no ECG changes
Implications of POCT in the Pre-hospital setting

Faster time to treatment:

Bringing POCT into the pre-hospital environment specifically for the measurement of potassium levels in hyperkalaemia will significantly benefit patients who have this prognosis and are not showing any signs, symptoms or related ECG changes. It will inevitably get these patients to the correct treatment faster as specific paramedics are able to give the required medications to reverse this prognosis.

Decrease cardiac death due to elevated potassium levels:

Hyperkalaemia is a reversible cause of cardiac arrest. Knowing the level of potassium using the POCT system may prove the diagnosis and the initiation of appropriate treatment can be started earlier.$$
\text { • }
$$
Advantages
- Evidence during a hyperkalemic cardiac arrest for the use of
reversible medications

- Diagnostic tool in the pre-hospital environment for long facilities with patients with no ECG changes.

- Beneficial for long inter-hospital transfers.

- Real time level of potassium and severity level can be determined (e.g. Mild, Moderate, Severe), therefore giving paramedics an understanding of what dose and treatment regime is appropriate.

POCT and the rapid availability of results is justified by long term societal gains including prolonged life and reduces hospital stays according to Laurence, Moss, Briggs, \& Beilby,
(2010, p. 165)

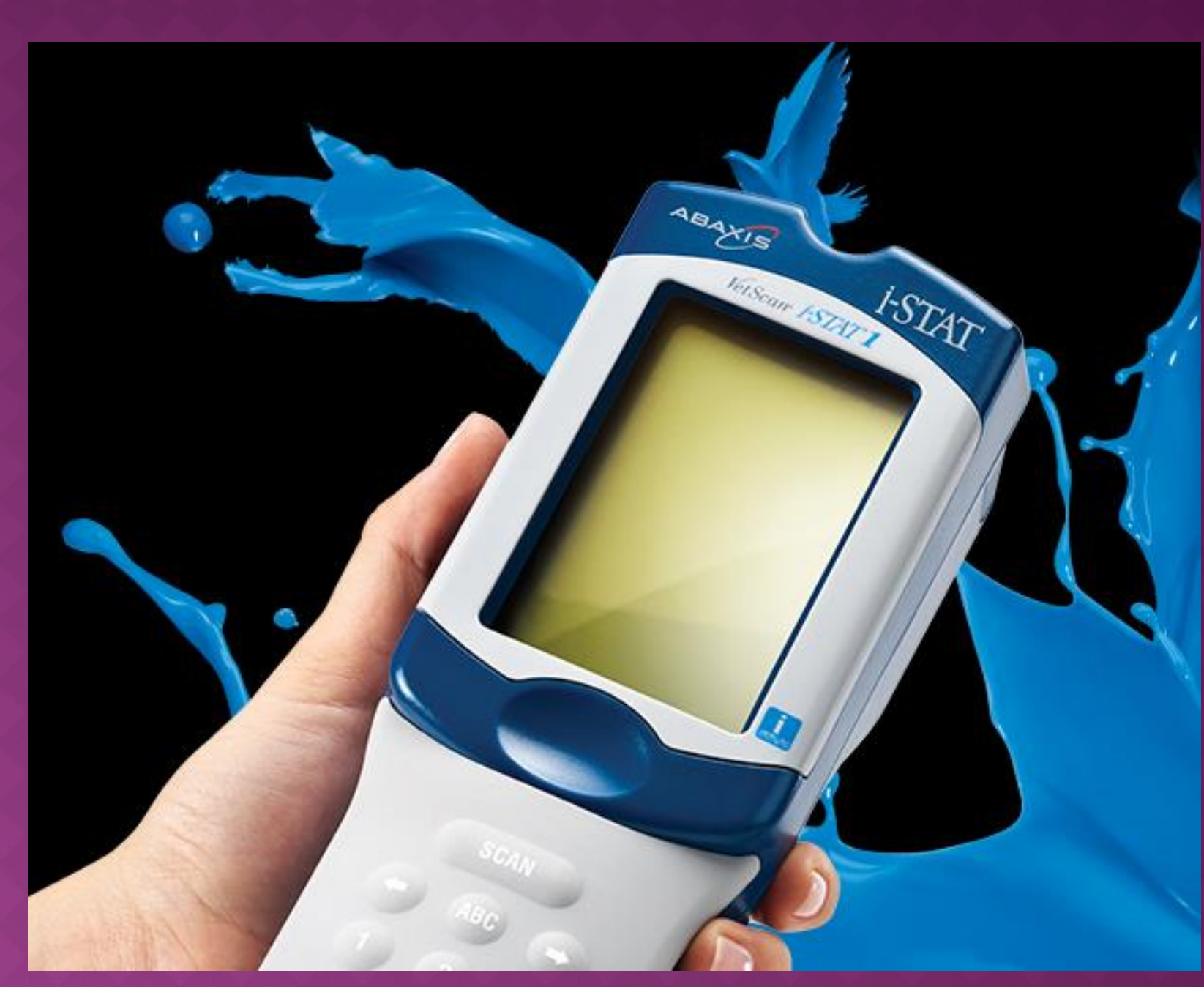

ISTAT Machine

- Portable

Produces lab accurate values for Blood Gases, Electrolytes, Chemistries Coagulation, Hematology, Gucose and Cardiac Markers (Troponins)

Only 2-3 drops of blood needed

$\$ 200$ per unit

Results in 2 minutes or less

- Allows faster diagnosis

\section{Disadvantages}

Requires extra training of staff across the board in normal ABG potassium levels and the use of the POCT equipment (Laurence, Moss, Briggs, \& Beilby, (2010, p. 174

The POCT unit itself can be expensive (price as above) 


\section{REFERENCES}

Ahn, S., Kim, W. Y., Sohn, C. H., Seo, D. W., Kim, W., \& Lim, K. S. (2011). Potassium values in cardiac arrest patients measured with a point-of-care blood gas analyzer. Resuscitation, 82(12), e25-e26. doi: http://dx.doi.org/10.1016/j.resuscitation.2011.08.010

Budak, Y. U., Huysal, K., \& Polat, M. (2012). Use of a blood gas analyzer and a laboratory autoanalyzer in routine practice to measure electrolytes in intensive care unit patients. $B M C$ anesthesiology, 12(1), 17.

Chacko, B., Peter, J. V., Patole, S., Fleming, J. J., \& Selvakumar, R. (2011). Electrolytes assessed by point-of-care testing-Are the values comparable with results obtained from the central

laboratory?. Indian journal of critical care medicine: peer-reviewed, official publication of indian Society of Critical Care Medicine, 15(1), 24.

EI-Sherif, N., \& Turitto, G. (2011). Electrolyte disorders and arrhythmogenesis. Cardiology $j$ ournal, 18(3), 233-245.

Fermann, G. J., \& Suyama, J. (2002). Point of care testing in the emergency department. J Emerg Med, 22(4), 393-404.

Khattak, H. K., Khalid, S., Manzoor, K., \& Stein, P. K. (2014). Recurrent life-threatening hyperkalemia without typical electrocardiographic changes. J Electrocardiol, 47(1), 95-97. doi: 10.1016/j.jelectrocard.2013.07.012

Laurence, C. O., Moss, J. R., Briggs, N. E., \& Beilby, J. J. (2010). The cost-effectiveness of point of care testing in a general practice setting: results from a randomised controlled trial. BMC Health Serv Res, 10, 165. doi: 10.1186/1472-6963-10-165

Graphics adapted from the texts above.

Picture received from:http://www.abbottpointofcare.com/Patient-Care-

Settings/Hospital/Critical-Care.aspx 


\title{
Adrenaline in Prehospital Cardiac Arrest
}

\author{
Rachel Kluck, $2^{\text {nd }}$ Year Paramedic Student, The University of Queensland
}

\section{BACKGROUND}

In the realm of prehospital cardiac arrest, adrenaline has been a mainstay of treatment for decades. Its administration is included in management protocols in emergency medical services throughout the world because of the drugs potent $\alpha$ and $\beta$ adrenergic stimulating properties that cause peripheral vasoconstriction and positive inotropic and chronotropic activity in the heart (Neumar et al., 2010). The American Medical Association's most recent guidelines still recommend a dosage schedule of $1 \mathrm{mg}$ every three to five minutes for both shockable and non-shockable rhythms (Neumar et al., 2010). However, the literature is lacking in evidence surrounding the effectiveness of adrenaline on mortality rates in out-ofhospital cardiac arrest patients.

\section{OBJECTIVES}

There are a number of aims that hope to be achieved, based around assessing the literature regarding various aspects of patient outcomes after adrenaline administration during cardiac arrest: - Prehospital return of spontaneous circulation (ROSC) - Patient mortality

- Neurological outcomes in cardiac arrest survivors

\section{METHODS}

A search of the literature was performed, with databases including PubMed and The Cochrane Library. Articles were chosen based on their coverage of the objectives. More recent articles were preferred (2005 onwards), but given the limited amount of research completed regarding this topic, using a timeframe that was too narrow would have greatly reduced the evidence available for analysis. Ideally, randomised controlled trials were sought, but many of the studies investigating these objectives were observational studies, often to prevent ethical dilemmas that arise in denying some subjects a potentially beneficial medication. For the same reason, study design was not taken into account during the research phase. Rather, study design and limitations was discussed in the findings so as to allow for a more complete assessment of the validity and relevance of the current literature surrounding the use of adrenaline in cardiac arrest. This method of research produced three articles addressing the outlined objectives.

Table 2: Cerebral Performance Category (CPC)

\begin{tabular}{|c|l|}
\hline CPC & \multicolumn{1}{|c|}{ Description } \\
\hline $\mathbf{1}$ & $\begin{array}{l}\text { Good cerebral performance: conscious, alert, able to work, might have mild neurologic or } \\
\text { psychological deficit. }\end{array}$ \\
\hline $\mathbf{2}$ & $\begin{array}{l}\text { Moderate cerebral disability: conscious, sufficient cerebral function for independent activities of } \\
\text { daily life. }\end{array}$ \\
\hline $\mathbf{3}$ & $\begin{array}{l}\text { Severe cerebral disability: conscious, dependent on others for daily support because of impaired } \\
\text { brain function. }\end{array}$ \\
\hline $\mathbf{4}$ & Coma or vegetative state: any degree of coma without the presence of all brain death criteria. \\
\hline $\mathbf{5}$ & Brain death: apnea, areflexia, EEG silence \\
\hline
\end{tabular}

\section{FINDINGS}

After analysing and bringing together the results from the three chosen articles, the overall conclusions around the impact of adrenaline on gaining ROSC, patient mortality and neurological function were similar. By examining the results of each study in isolation, as well as their respective study design and limitations, a By examining the results of each study in isolation, as well as their respective s
better conclusion could be drawn on the collective viewpoint of the literature.

Jacobs and Finn et al conducted the first randomised controlled trial (RCT) comparing clinical outcomes for patients who received adrenaline versus a placebo during cardiac arrest. It was a double-blind placebo controlled study, conducted in Western Australia between 2006 and 2009. According to their results, adrenaline significantly increases that rate at which prehospital ROSC is obtained compared to placebo, at $23.5 \%$ and $8.4 \%$ respectively (Table 1) (Jacobs et al., 2011), with this pattern reflected in hospital admission rates of $25.4 \%$ and $13 \%$ respectively. However, in their secondary outcomes of survival to discharge, there was not a significant difference between those who received adrenaline and those that did not. In addition, neurological outcomes in those who survived to dischare - measured as per the Cerebrat Performance neurological outcomes in those who survived to discharge - measured as per the Cerebral Performanc Category (CPC) (Table 2) - did not exhibit a significant difference (Table 1) (Jacobs et al., 2011). Despite this
study providing some prominent results, there were some design problems with the study. While all study providing some prominent results, there were some design problems with the study. While all
paramedics were governed by Australian Resuscitation Guidelines and did not administer any other paramedics were govened by Austral,an Resuscitation Guidelines and did not administer any other resuscitation drugs during the trial, the study itself was underpowered to detect significant outcomes. The trial was initially calculated to require 2213 individuals per treatment group, which was originally obtainable because several other ambulance services were intending to participate. However, these other services withdrew from the trial, and since participation was voluntary, only $44 \%$ of paramedics took part (Jacobs et al., 2011). Consequently, less than 300 individuals were placed in each treatment group, significantly fewer than the number required to give the study a power of at least $80 \%$. As such, the study provided some significant results as the first conducted RCT investigating adrenaline administration during cardiac arrest, but had some major methodological issues.

An observational study undertaken in Japan in 2005-2008 (Atiksawedparit et al., 2014) looked at nearidentical outcomes as in Western Australia with similar results, all at $p<0.0001$. In the group who received adrenaline versus those who did not receive adrenaline, the rate at which prehospital ROSC was achieved was $18.3 \%$ and $10.5 \%$ respectively (Hagihara et al., 2012) (Table 1). At one month post-arrest, survival rates between groups were $5.1 \%$ and $7 \%$ respectively (Hagihara et al., 2012). In term of neurological outcomes, $1.3 \%$ of patients in the adrenaline group had a CPC score of 1 or 2 , while $3.1 \%$ of those who did not receive adrenaline achieved this neurological outcome (Hagihara et al., 2012) (Table 1). As such, the results from this study suggest that adrenaline administration during cardiac arrest improves the chance of obtainin ROSC, but had unfavourable outcomes when considering one-month mortality and neurological status.
This study had advantages in that because it was observational, it had a large participation rate, with 13401 individuals in each treatment group (Hagihara et al., 2012) and a power of $94.1 \%$. However, this study had a number of limitations. In the Japanese EMS system, EMS personnel only became permitted to administer adrenaline part way through the study, before which consultation with a physician was required (Hagihara et al., 2012). Physician presence in ambulances was highly variable, and automated external defibrillators had only been introduced prehospitally a few years prior to the study, meaning the Japanese EMS system was still relatively inexperienced in modern cardiac arrest management. In addition, clinicians were not blinded and inhospital treatment was not recorded, adding potential confounders to the results.

Table 1: Summary of Results from Chosen Studies

\begin{tabular}{|c|c|c|c|c|c|c|c|c|c|c|}
\hline \multirow{3}{*}{\multicolumn{2}{|c|}{ Author }} & \multicolumn{9}{|c|}{ Patient Outcome } \\
\hline & & \multicolumn{3}{|c|}{ Prehospital ROSC (\%) } & \multicolumn{3}{|c|}{$\begin{array}{c}\text { 1-Month Survival / Survival } \\
\text { to Discharge (\%) }\end{array}$} & \multicolumn{3}{|c|}{ CPC Score 1 or $2(\%)$} \\
\hline & & Adrenaline & \begin{tabular}{|c|c|} 
No \\
Adrenaline
\end{tabular} & P-Value & Adrenaline & $\mid \begin{array}{c}\text { No } \\
\text { Adrenaline }\end{array}$ & P-Value & Adrenaline & $=\mid$\begin{tabular}{c|} 
No \\
Adrenaline
\end{tabular} & P-Value \\
\hline \multicolumn{2}{|c|}{ Jacobs et al } & 23.5 & 8.4 & $<0.001$ & 4.0 & 1.9 & NS & 81.8 & 100 & NS \\
\hline \multicolumn{2}{|c|}{ Hagihara et al } & 18.3 & 10.5 & $<0.001$ & 5.1 & 7.0 & $<0.001$ & 1.3 & 3.1 & $<0.001$ \\
\hline \multirow{2}{*}{$\begin{array}{l}\text { Gotc } \\
\text { et al }\end{array}$} & $s$ & 22.8 & 27.7 & $<0.0001$ & 15.4 & 27.0 & $<0.0001$ & 7.0 & 18.6 & $<0.0001$ \\
\hline & N.S. & 18.7 & 3 & $<0.0001$ & 3.9 & 2.2 & $<0.0001$ & 0.59 & 0.62 & NS \\
\hline
\end{tabular}

The third article chosen to review with regards to the major objective was another observational study also in Japan, but during the 2009-2010 period (Atiksawedparit et al., 2014). Their results were segregated into those for shockable and non-shockable rhythms. For shockable rhythms, ROSC rates were lower in the adrenaline group and than for those who did not receive adrenaline, at $22.8 \%$ and $27.7 \%$ respectively (Goto \& Maeda, 2013) (Table 1). One-month survival rates were $15.4 \%$ and $27 \%$ respectively, and the rate of achieving CPC 1 or 2 in the adrenaline group versus the no-adrenaline group was $7 \%$ and $18.6 \%$. All of these results were at a high level of significance, with $p<0.0001$ (Goto \& Maeda, 2013). In non-shockable cardiac arrests, prehospital ROSC achievement was $18.7 \%$ in the adrenaline group and $3 \%$ for those who did not receive adrenaline (Table 1 ). Similarly, one-month survival was higher in the adrenaline group, at $3.9 \%$ and $2.2 \%$ respectively, $p<0.0001$ (Goto \& Maeda, 2013). While these results were also at a high level of significance, there was not a significant correlation found in terms of neurological outcomes (Table 1). Again, a number of limitations were present with this study. Patients were not randomised, and adrenaline was only indicated (as per FDMA guidelines) for non-shockable rhythms that were refractory to compressions or non-shockable rhythms that became so after defibrillation of a shockable rhythm (Goto \& Maeda, 2013). These criteria may have biased the adrenaline patients to worse outcomes. In addition, there was no analysis done regarding the number of doses and their associated outcomes, and in-hospital management was not followed up. However, despite these methodological issues, the study raises a few key points in that perhaps the effectiveness of adrenaline should be assessed in terms of the presenting rhythm. This study found that adrenaline was beneficial in shockable rhythms but not non-shockable rhythms. Different pathologies may respond differently to the adrenergic properties of adrenaline and this warrants further investigation.

\section{CONCLUSIONS}

Across the three studies discussed, there was a similar trend exhibited in the results. Generally, adrenaline increased that rates at which prehospital ROSC was achieved, but was associated with equal or decreased rates of good neurological outcomes and one-month month survival. The exception was in Goto and Maeda's observational study that exhibited increased one-month survival rates when adrenaline was administered to patients presenting with a non-shockable rhythm. All studies had limitations and methodological problems that prevent the integration of these results into Advanced Cardiovascular Life Support (ACLS) Guidelines. This review of recent studies regarding this topic has allowed for a number of recommendations to be formed for consideration during the planning and execution of future trials:

- Utilising double-blind, placebo-controlled randomised controlled trials will minimise selection bias and inconsistent drug administration

Recording and analysing the additional relationship between the number of doses administered, in-hospital treatment and patient outcomes

Conduct study in a location where clinician skill levels are capable of performing current ALCS guidelines

Use multi-centre approach to ensure participant numbers are high enough to give the study an appropriate level of power

Although strong and consistent evidence of the effects of adrenaline on patient outcomes after cardiac arrest is not currently available, the literature has provided a basis for future research. Implementation of these recommendations forthcoming studies will assist in establishing a reliable platform on which to base future cardiac arrest management guidelines.

References:

Atiksawedparit, P., Rattanasiri, S., McEvoy, M., Graham, C.A., Sittichanbuncha, Y., \& Thakkinstian, A. (2014). Effects of
prehospital adrenaline administration on out-of-hospital cardiac arrest outcomes: a systematic review and meta-analysis. prehospital adrenaline administration on out-of-hospital cardiac arrest outcomes: a systematic review and meta-analysis.
Critical Care, 18(463), 1-12. Goto, Y., Maeda, T., \& Goto, Y.N. (2013). Effects of prehospital epinephrine during out-of-hospital cardiac arrest with initial non-shockable rhythm: and observational cohort study. Critical Care, 17, 1-11. Hagihara, A., Hasegawa, M., Abe, T., Nagata, T., Wakata, Y., \& Miyazaki, S. (2012). Prehospital epinephrine use and survival
among patients with out-of-hospital cardiac arrest. Journal of the American Medical Association, 307(11), 1161-1168.

Jacobs, I.G., Finn, J.C., Jelinek, G.A., Oxer, H.F., \& Thompson, P.L. (2011). Effect of adrenaline
cardiac arrest: A randomised double-blind placebo-controlled trial. Resuscitation, $82,1138-1143$.

Neumar, R.W. et al. (2010). Part 8: adult advanced cardiovascular life support: 2010 American Heart Association guidelines for
cardiopulmonary resuscitation and emergency cardiovascular care. Circulation, 122, S729-S767. US Food and Drug Administration. (2014). Cerebral Performance Categories Scale. Retrieved from http://www.fda.gov/ohrms/ dockets/ac/05/briefing/2005-4100b1_03_CPC\%20Scale.pdf 


\title{
What do we know about crowdsourcing the Chain of Survival using mobile phones?
}

\author{
David Still, Australian Catholic University, Canberra.
}

Objective: To review the available research on the use of mobile phones to support the chain of survival by dispatching laypersons to out-of-hospital cardiac arrests.

Background: The chain of survival has been central to out-of-hospital cardiac arrest (OHCA) management since the early 1990s (1). Several solutions for 'crowdsourcing' the chain of survival (ie, dispatching laypersons to cardiac arrests) have used mobile phone messaging and apps, some of which have seen rapid public uptake (2). However, their role in the chain of survival has only recently been researched.

Methods: MeSH-compatible search terms were identified, and a search performed using CINAHL, Embase, Pubmed, Science Direct, ProQuest and Google Scholar. 96 articles relating to the use of mobile phones in out-of-hospital cardiac arrest were identified. In the included articles, 9 studied the use of mobile phones to dispatch laypersons to cardiac arrests while 2 others studied AED-mapping apps. 1 was excluded as a duplicate, and 5 articles were only available as abstracts. Results relating to the chain of survival were then tabulated and compared against the goals of early access, early CPR, and early defibrillation.

Results: The included articles studied crowdsourcing solutions that were administered by the local emergency medical services (EMS), using either text message dispatch or a smartphone app. No articles compared these two solutions directly. No articles included solutions that operate without EMS involvement (eg, Good SAM).

No articles reported a primary outcome of patient survival or neurological status. 89 other findings were reported, of which 10 were reported by more than one article.

\begin{tabular}{|c|c|c|}
\hline Step & Outcome & Result \\
\hline \multirow{5}{*}{ 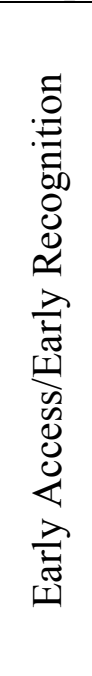 } & User Profession & $\begin{array}{l}\text { PulsePoint: Firefighters ( } 30 \%) \text {, Paramedics } \\
(21 \%) \text {, EMT (16\%), Nurse }(4.4 \%), \text { MD } \\
(0.83 \%)(3) \\
\text { AED-Alert: "One-third" involved with } \\
\text { police, fire or medicine (4) }\end{array}$ \\
\hline & $\begin{array}{l}\text { Dispatch time (only measuring } \\
\text { data input time by EMS) }\end{array}$ & Motegi: 37 seconds (range 21-60) (5) \\
\hline & $\begin{array}{l}\text { Total dispatch time (incl. data } \\
\text { input and geolocation) }\end{array}$ & $\begin{array}{l}\text { Mobile Life Saver: } 87 \text { seconds (SD } \pm 45 \mathrm{sec} \text {; } \\
\text { simulation) (6) } \\
\left.\text { Bucharest }^{1} \text { : "A few seconds" ( } 7\right)\end{array}$ \\
\hline & $\begin{array}{l}\text { Proportion of laypersons } \\
\text { responding to alerts }\end{array}$ & $\begin{array}{l}\text { PulsePoint: } 23 \%(\mathrm{n}=157)(3) \\
\text { AED-Alert: } 28.2 \%(\mathrm{n}=579)(4)\end{array}$ \\
\hline & $\begin{array}{l}\text { Time reduction to arrival } \\
\text { (compared to EMS) }\end{array}$ & $\begin{array}{l}\text { Motegi: } 127 \text { seconds (simulation, rural } \\
\text { setting; trial arm actual response time: } 6 \text { min }\end{array}$ \\
\hline
\end{tabular}

\footnotetext{
${ }^{1}$ Where the name of the service is unavailable, its location is given instead.
} 


\begin{tabular}{|c|c|c|}
\hline & & $\begin{array}{l}21 \mathrm{sec})(5) \\
\text { Mobile Life Saver: } 140 \text { seconds (simulation, } \\
\text { urban setting) }(6)\end{array}$ \\
\hline \multirow{3}{*}{ 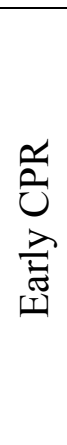 } & $\begin{array}{l}\text { Time from dispatch to CPR } \\
\text { commencement }\end{array}$ & Bucharest: "1-3 minutes" (7) \\
\hline & $\begin{array}{l}\text { Frequency of CPR } \\
\text { commencement by layperson- } \\
\text { responders when indicated }\end{array}$ & $\begin{array}{l}\text { PulsePoint: } 80 \%(n=8)(3) \\
\text { AED-Alert: } 49.3 \%(n=37)(4) \\
\text { Mobile Life Saver: } 30 \%(n=6)(6)\end{array}$ \\
\hline & $\begin{array}{l}\text { Total number of patients who } \\
\text { received early CPR }\end{array}$ & AED-Alert: $n=11(4)$ \\
\hline \multirow{3}{*}{ 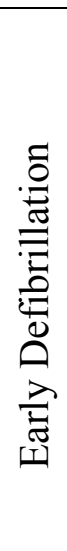 } & $\begin{array}{c}\text { Frequency of AED connection } \\
\text { in } \leq 6 \text { minutes (from time of } \\
\text { emergency call) }\end{array}$ & TM-Alert: $16.5 \%(\mathrm{n}=29)(8)$ \\
\hline & Frequency of early defibrillation & $\begin{array}{l}\text { AED-Alert: } \mathrm{n}=7 \text { ("early defibrillation") (4) } \\
\text { TM-Alert: } 10.5 \%(\mathrm{n}=8) \text { ("first shock in } \leq 6 \\
\text { minutes") (8) }\end{array}$ \\
\hline & $\begin{array}{l}\text { Time reduction to first shock } \\
\text { (compared to EMS) }\end{array}$ & TM-Alert: $2 \min 39 \sec (8)$ \\
\hline
\end{tabular}

Discussion: No trials have attempted to assess the effect of 'crowdsourced' CPR on patient mortality, return of spontaneous circulation, or haemodynamic parameters, which are the preferred outcomes for smaller trials of cardiac arrest interventions (9). The included papers reported a very wide range of outcomes to do with processes, such as the response times and travel distances, user actions and demographics.

The evidence suggested that the studied solutions were an effective part of the chain of survival. Merchant et al found that AED mapping could be crowdsourced cheaply and accurately (10), while the responder apps were found to significantly reduce response time, time to CPR and time to defibrillation. One simulation found that only response distance was reduced, without reducing response time. Only two papers assessed adverse effects: one paper reported none (6), while another found that $2 \%$ $(n=12)$ of laypersons required follow-up care (4).

Comparison between solutions is difficult. On the available evidence, it is not possible to assess the relative merits of text messages sent to users' mobile phones versus in-app notifications using smartphones, or to compare dispatch processes initiated by the local EMS versus those initiated directly by bystanders.

Conclusion: Mobile phones are likely to be useful for crowdsourcing the chain of survival to reduce the time to access, cardiopulmonary resuscitation and defibrillation. There is no evidence for the superiority of any particular dispatch method or strategy. 


\section{References:}

1. American Heart Association. Improving Survival From Sudden Cardiac Arrest: The "Chain of Survival" Concept. Circulation. 1991 May; 83(5): p. 1832-1847.

2. Brooks S. Abstract 191: Community Uptake of a Smartphone Application to Recruit Bystander Basic Life Support for Victims of Out-of-Hospital Cardiac Arrest. Circulation. 2012;(126): p. A191.

3. Brooks S, Worthington H, Gonedalles T, Bobrow B, Morrison L. Implementation of the PulsePoint smartphone application for crowd-sourcing bystander resuscitation. Critical Care. 2014; 18(Suppl 1): p. S176.

4. Scholten A, van Manen J, van der Worp W, Ijzerman M, Doggen C. Early cardiopulmonary resuscitation and use of Automated External Defibrillators by laypersons in out-of-hospital cardiac arrest using an SMS alert service. Resuscitation. 2011;(82): p. 1273-1278.

5. Yonekawa C, Suzukawa M, Yamashita K, Kubota K, Yasuda Y, Kobayashi A, et al. Development of a first-responder dispatch system using a smartphone. Journal of Telemedicine and Telecare. 2014; 20(2): p. 75-81.

6. Ringh M, Fredman D, Nordberg P, Stark T, Hollenberg. Mobile phone technology identifies and recruits trained citizens to perform CPR on out-of-hospital cardiac arrest victims prior to ambulance arrival. Resuscitation. 2011; 82(12).

7. Grasu A. SMS alerts for volunteer rescuers in the emergency system: Decreasing the delay in starting cardiopulmonary resuscitation [abstract]. Resuscitation. 2013; 81 (Suppl 1).

8. Zijlstra J, Stieglis R, Riedijk F, Smeekes M, van der Worp W, Koster R. Local lay rescuers with AEDs, alerted by text messages, contribute to early defibrillation in a Dutch out-of-hospital cardiac arrest dispatch system. Resuscitation. 2014.

9. American Heart Association. Primary Outcomes for Resuscitation Science Studies: A Consensus Statement From the American Heart Association. Circulation. 2011 (124): p. 2158-2177.

10. Merchant, R., Asch, D., Hershey, J., Griffis, H., Hill, S., et al. A Crowdsourcing Innovation Challenge to Locate and Map Automated External Defibrillators. Circulation: Cardiovascular Quality and Outcomes. 2013 March, 6(2): p. 229236. DOI: 10.1161/CIRCOUTCOMES.113.000140

11. Sakai T, Iwami T, Kitamura T, Nishiyama C, Kawamura T, Kajino K, et al. Effectiveness of the new 'Mobile AED Map' to find and retrieve an AED: A randomised controlled trial. Resuscitation. 2011; 82(1): p. 69-73 


\section{Socioeconomic Status and its effect on pregnancy and childbirth: paramedic considerations in the prehospital setting}




\begin{abstract}
This article aims to identify the role of socioeconomic status in determining poor health outcomes in pregnancy and childbirth. It brings to light the limitations and complications that a person in a low socioeconomic society may face and the effect that this possibly has on the health of the mother and child. The literature has been reviewed in relation to three key areas, namely: social and emotional matters, lifestyle factors and financial issues. Particular focus has been put on understanding these issues from a paramedic perspective and how this can assist in both treating and educating the patient in the pre-hospital environment. While there has been sufficient research into the individual areas of socioeconomic status affecting pregnancy, this article has drawn these topics together to create an overview of a subject which is complex and multifaceted.
\end{abstract}

\title{
KEY WORDS
}

pregnancy; low socioeconomic status; paramedics; drug and alcohol abuse; financial issues; stress and support; poor health outcomes; birth defects

\section{INTRODUCTION}

Low socioeconomic status (SES) has long been associated with poor health outcomes in the developed world when compared against high SES in the same vicinities. This is an important consideration for paramedics in comprehending their environment and understanding cause and effect. It is relevant therefore to explore the health discrepancies between low SES and high SES communities from the same regions and how this affects specific issues in the community. This article will explore the relationship between SES and poor health outcomes in pregnancy and childbirth focusing specifically on the prehospital aspect. Socioeconomic disadvantage and its effect on health is a multifaceted issue however this article will attempt to focus on three of the key aspects of low SES communities: social and emotional matters, lifestyle choices and financial issues. It will identify the negative impact on both the mother and baby and outline considerations which paramedics must keep in mind when attending pregnancy and childbirth related jobs in low SES communities.

\section{SOCIAL AND EMOTIONAL ISSUES}

\section{Stress and Lack of Support}

Females in low SES societies are already under increased stress and this is exacerbated by the extra stressors of pregnancy. The extra stress that low SES people are put under can be attributed to financial troubles, fear for safety and lack of support just to name a few. (1) In addition, during pregnancy there is extra pressure put on the mother as there is increased financial struggle due to the inability to work as well as the anticipation of not coping when the baby arrives. (1) It has also been shown that people in low SES societies have a lower ability to cope with stress possibly due to lack of education and life experience. (2)

\section{Support}

Support is proven to have a positive impact on pregnancy whether it be from the father of the child, grandparents, friends or a community nurse.(3) It has been shown that greater paternal involvement and support during pregnancy results in lower anxiety levels in pregnant women.(4) Due to the often unsafe and unstable surroundings which a low SES female is faced with, they may find it hard to put their trust in 
someone during such a vulnerable time.(5) Females in low SES communities often have smaller social groups and strain on such friendships is increased during pregnancy, especially if the expectant mother is relying heavily on social support rather than family.(1) Functional family relationships are helpful in providing help and support to the expectant mother however they are rare in low SES societies.(3)

\section{Stress}

Stress during pregnancy can be linked to poor health outcomes such as anxiety and depression (6)(7) and is believed to be connected with the foetus via the corticosteroid pathway.(8) Extra stressors put on expectant mothers during a time when they are already susceptible to mental illness can have an extremely negative impact.(5) Paramedics should understand that lower SES females are likely to be depressed, anxious or facing other mental illnesses.(6) In extreme cases, preterm birth can also result from stress during pregnancy.(3) Paramedics must consider the increased risk of preterm labour and birth when attending cases that are in low SES communities.(9) Preterm birth is the birth of a child prior to 37 weeks gestation and depending on the gestational age of the baby, different outcomes can occur.(10) There is a higher neonatal morbidity and mortality in lower SES groups which can be linked to higher rates of preterm birth.(8) It is important to get a good history from the patient and to evaluate the risk of a preterm birth, understanding the complications that are associated.

\section{LIFESTYLE CHOICES}

\section{Smoking}

It is no secret that there is a higher prevalence of smoking in lower SES societies than high SES ones.(11) Due to the increasing evidence linking smoking and adverse health outcomes, the effect of smoking on health and morbidity is a well-researched topic. However the relationship between smoking during pregnancy and harmful consequences for both the baby and mother are not as widely researched. In 2002/2003, 18 percent of pregnant females smoked during pregnancy. (12) High smoking rates in low SES communities can be attributed to lack of education (12) and peer pressure.(5) Not only are low SES females more likely to smoke, they are also less likely to seek help from doctors to quit.(11) Low SES females are often unaware of pregnancy until late into the first trimester and may only begin to cease or reduce smoking habits when pregnancy is confirmed.(12)

Even a small number of cigarettes consumed during conception and pregnancy can be detrimental (13) and can increase the risk of preterm birth, a low birth-weight, placenta previa and placental abruption.(12) Females who smoke during pregnancy have a 30\% higher chance of giving birth prematurely.(14) In addition, smoking during pregnancy increases the risk of sudden infant death syndrome.(14) It has also been linked to but not proven with enough supporting evidence that smoking during pregnancy causes cleft palate, reduced respiratory function in the newborn and cognition difficulties in childhood.(14) In the prehospital environment, paramedics must recognise the increased risk of these conditions when attending a patient in a low SES society. While it does not change the treatment or interventions, it is still important to be able to identify these issues early and take the appropriate action.

\section{Recreational drug and alcohol abuse}

Recreational drug and alcohol abuse is common in low SES communities. Alcohol and drug use during pregnancy is linked to poor health outcomes for both the mother and the baby.(15) In 2002/2003, 4.3 percent of pregnant females used illicit drugs and 4.1 percent took part in binge alcohol consumption.(12) Due to poor education, low self-esteem, lack of contraceptive agents such as the contraceptive pill and reduced rate of abortions, females in the 15-25 year old age bracket in low SES societies are more likely to fall pregnant and continue to use illicit drugs and alcohol.(12) As with smoking, those who use drugs and alcohol are not likely to tell their doctors as there is stigma surrounding this topic.(11) Given the range of 
social drugs on the market, it is impossible to understand the effect that each drug can have and their short and long term effects on pregnancy and childbirth.(14) Paramedics are therefore required to be flexible and open to a range of possibilities and able to act accordingly.

\section{Alcohol}

Alcohol abuse before falling pregnant can cause health deficits in the mother such as pancreatitis, cardiomyopathy, coagulopathy and malnutrition just to name a few.(15) This already creates an unhealthy environment for a foetus to grow, regardless of alcohol consumption levels during pregnancy.(15) This, however, is not the main concern related to alcohol abuse and pregnancy. Ethanol easily crosses the placental barrier and this means that alcohol consumption by the mother directly affects the growing foetus during pregnancy.(15) If alcohol is consumed during pregnancy, the newborn may suffer from foetal alcohol syndrome (FAS).(14) This syndrome has a number of symptoms including but not limited to mental handicap, musculoskeletal, cardiovascular and genitourinary abnormalities.(15) Binge drinking during pregnancy can account for 18 percent of neonatal mortalities.(15) Some other foetal health concerns include microcephaly and other growth defects.(12)

\section{Narcotics}

Narcotic use can have an indirect (poor maternal health) and direct (transplacental opiod transfer) effect on the foetus.(15) It has been linked to perinatal mortality, preterm delivery and preterm rupture of membranes.(12) Unlike smoking and alcohol, it is not recommended that opiates are completely ceased during pregnancy as this can result in withdrawal symptoms that can be detrimental to the baby.(14)(15) Rather than completely stopping opiate consumption during pregnancy, it is recommended that methadone be substituted.(14) For this reason, it is important for expectant mothers to seek medical help rather than trying to quit on their own.(15) If the pregnant female reaches the stages of labour and needs opioid substitutes (methadone) in labour, it is important that paramedics take them to an appropriate facility. It is also important for paramedics to understand the signs of withdrawal and how this can affect both the mother and the unborn child. Babies can be born with respiratory depression due to the transplacental effect of maternal narcotic consumption (14) and this may have to be managed in the prehospital environment. Severe withdrawal symptoms of the newborn are commonly seen within the first 24-72 hours of birth and can include tremors, seizures and respiratory distress just to name a few.(14) While most symptoms present within this period, it is also possible for the effects to occur 10-36 days post birth.(14) These are all things that paramedics must consider when attending cases in low SES communities which are associated with pregnancy, childbirth and infants.

\section{CNS Stimulants}

Other drugs such as central nervous system (CNS) stimulants (cocaine, amphetamines) can also directly and indirectly affect the foetus. These drugs are more associated with long-term effects such as cognition and behavioural deficits however can still increase the risk of spontaneous abortion, placental abruption and growth restrictions.(12) It has been found that the indirect effect of cocaine abuse is a result of the mother's vasoconstriction and therefore reduced uteroplacental blood flow.(15) Indirect effects of CNS stimulants are most likely the cause of adverse health outcomes for the baby as these drugs cause hypertension, arrhythmias and myocardial ischaemia in the expectant mother.(15) The use of anaesthetics should be done so with caution in cocaine addicted patients as it may cause complications.(15)

\section{Poor diet}

Lifestyle choices such as poor diet stem from childhood and are continued throughout a person's life, including pregnancy.(5). An adequate diet and healthy lifestyle is paramount in creating the basis for a healthy baby.(16) People in low SES communities often consume diets that are low in key nutrients and 
high in saturated fat.(5) Poor diets have been associated with poor foetal growth, preterm delivery and an increased risk of chronic diseases later in life.(16) They also can increase the risk of gestational diabetes and pre-eclampsia in the expectant mother.(16) While not in the forefront of causes of ambulance call-outs during pregnancy, they are still things that a paramedic must take into account when treating a low SES pregnant patient.

\section{FINANCIAL ISSUES}

\section{Cost of medical assistance and antenatal care}

Those who have low socioeconomic status are financially unstable and this can be linked to poor health outcomes in pregnancy and everyday life. While there is little research on the specific effect of limited money and pregnancy outcomes, it is well known that medical costs are quite significant in any setting. General practitioner (GP) visits, ante-natal care, birthing classes and private hospitals can cost a substantial amount of money, especially if you take things such as travel to and from appointments and missing work into consideration. Studies have shown that there is a relationship between attending antenatal care classes and a reduced incidence of preterm birth.(17) However that relationship is proportional to the number of classes attended.(17) In addition, rates of oral contraceptives such as the contraceptive pill are lower in low SES communities due to their cost.(18) This results in more unwanted pregnancies, less abortions and a higher rate of pelvic inflammatory disease which in turn increases the rate of ectopic pregnancies. (18)

\section{Environmental factors}

Low SES communities are situated in areas of high pollutant exposure due to the lower cost of living in these areas.(19) Air pollutants which can be found in and around the home as well as at work can increase the risk of a small birth weight or preterm birth at high concentrations.(20) Other pollutants such as lead and mercury can be found in batteries, pottery glazes and paints and can also lead to poor health outcome.(21) There are few studies that look at the direct link between low SES and high incidence of pollutant toxicity and its association with pregnancy.

\section{CONCLUSION}

This article has only scratched the surface of socioeconomic status and its effect on pregnancy and childbirth. It has, however, brought to light the major issues that low SES females face which result in poor health outcomes during pregnancy and childbirth. While there are certain areas surrounding this topic which need to be further studied, overall the topic has been well researched in the community. Paramedics are at the forefront of both pre-hospital health care and patient education on how they can reduce poor health outcomes especially during pregnancy and childbirth. While it is not an issue that will be quickly resolved, it is important for paramedics to understand their role in improving patient outcomes. 


\section{REFERENCES}

1. Byrd-Craven J, Massey A. Lean on me: Effects of social support on low socioeconomic-status pregnant women. Nursing \& health sciences. 2013; 15 (3): 374-378.

2. Andrews L. Encyclopedia of depression. Santa Barbara, Calif.: Greenwood Press; 2010.

3. Shapiro G, Fraser W, Frasch M, S 'Eguin J. Psychosocial stress in pregnancy and preterm birth: associations and mechanisms. Journal of perinatal medicine. 2013; 41 (6): 631-645.

4. Zachariah R. Social support, life stress, and anxiety as predictors of pregnancy complications in lowincome women. Research in nursing \& health. 2009; 32 (4): 391-404.

5. Chen E, Miller G. Socioeconomic status and health: mediating and moderating factors. Annual review of clinical psychology. 2013; 9: 723-749.

6. Miech R, Caspi A, Moffitt T, Wright B, Silva P. Low socioeconomic status and mental disorders: a longitudinal study of selection and causation during young adulthood. American Journal of Sociology. 1999; 104 (4): 1096-1131.

7. Christie A, Barling J. Disentangling the indirect links between socioeconomic status and health: The dynamic roles of work stressors and personal control.. Journal of Applied Psychology. 2009; 94 (6): 1466.

8. Ruth C, Roos N, Hildes-Ripstein E, Brownell M. The influence of gestational age and socioeconomic status on neonatal outcomes in late preterm and early term gestation: a population based study. $B M C$ pregnancy and childbirth. 2012; 12 (1): 62.

9. Woollard M. Pre-hospital obstetric emergency training. Chichester, West Sussex, UK: Wiley-Blackwell; 2010 .

10. Whitehead N. The relationship of socioeconomic status to preterm contractions and preterm delivery. Maternal and child health journal. 2012; 16 (8): 1645--1656.

11. Cnattingius S. The epidemiology of smoking during pregnancy: smoking prevalence, maternal characteristics, and pregnancy outcomes. Nicotine \& Tobacco Research. 2004; 6 (Suppl 2): 125-140.

12. Rayburn W. Alcohol and substance abuse. In: Queenan J, Spong C, Lockwood C. (eds.)Management of High-Risk Pregnancy: an evidence-based approach. 5th ed. Massachusetts: Blackwell Publishing; 2007. pp. 24-30.

13. Al-Sahab B, Saqib M, Hauser G, Tamim H. Prevalence of smoking during pregnancy and associated risk factors among Canadian women: a national survey. BMC pregnancy and childbirth. 2010; 10 (1): 24.

14. Schaefer C, Peters P, Miller R. Drugs during pregnancy and lactation. Amsterdam: Elsevier; 2007.

15. Kuczkowski K. The effects of drug abuse on pregnancy. Current Opinion in Obstetrics and Gynecology. 2007; 19 (6): 578--585.

16. Ahmed F, Tseng M. Diet and nutritional status during pregnancy. Public health nutrition. 2013; 16 (08): 1337-1339.

17. Beeckman K, Louckx F, Downe S, Putman K. The relationship between antenatal care and preterm birth: the importance of content of care. The European Journal of Public Health. 2013; 23 (3): 366-371. 
18. Yuk J, Kim Y, Hur J, Shin J. Association between socioeconomic status and ectopic pregnancy rate in the Republic of Korea. International Journal of Gynecology |\& Obstetrics. 2013; 122 (2): 104-107.

19. Evans G, Kantrowitz E. Socioeconomic status and health: the potential role of environmental risk exposure. Annual Review of Public Health. 2002; 23 (1): 303-331.

20. Nieuwenhuijsen M, Dadv, Grellier J, Martinez D, Vrijheid M, Others. Environmental risk factors of pregnancy outcomes: a summary of recent meta-analyses of epidemiological studies. Environ Health. 2013; 12: 6 .

21. Goetzl L. Environmental agents and reproductive risk. In: Queenan J, Spong C, Lockwood C. (eds.) Management of High-Risk Pregnancy: an evidence-based approach. 5th ed. Massachusetts: Blackwell Publishing; 2007. pp. 31-35. 